\title{
Constipação em lactentes: influência do tipo de aleitamento e da ingestão de fibra alimentar
}

\author{
Constipation in infants: influence of type of feeding and dietary fiber intake \\ Andrea Nogueira de Campos Aguirre1, Márcia Regina Vitolo², \\ Rosana Fiorini Puccini ${ }^{3}$, Mauro Batista de Morais ${ }^{4}$
}

\begin{abstract}
Resumo
Objetivo: estudar a associação entre tipo de aleitamento, consumo de fibra alimentar e ocorrência de constipação em lactentes.

Métodos: foram estudados 275 lactentes atendidos consecutivamente em unidade básica de saúde. O tipo de aleitamento foi classificado como predominante, misto e artificial. Constipação foi caracterizada pela eliminação de fezes duras, associada a uma das seguintes características: dor ou dificuldade ao evacuar, fezes em cíbalos ou cilíndrica, com rachaduras, e intervalo entre as evacuações maior ou igual a 3 dias. Pseudoconstipação foi caracterizada quando ocorria a eliminação de fezes amolecidas, sem dor ou dificuldade, em intervalo maior ou igual a 3 dias.

Resultados: constipação foi observada em $25,1 \%$ dos lactentes. Pseudoconstipação ocorreu exclusivamente nos primeiros 6 meses de vida, em $5,1 \%$ dos 159 lactentes desta faixa etária. Constipação entre os 6 e 24 meses $(38,8 \% ; 45 / 116)$ foi mais freqüente do que no primeiro semestre de vida $(15,1 \%, \mathrm{p}=0,0000)$. A análise de regressão logística evidenciou que, no primeiro semestre, os lactentes em aleitamento artificial demonstram chance 4,5 vezes maior de apresentar constipação do que os em aleitamento predominante. Entre os de 6 a 24 meses, a estimativa de consumo de fibra alimentar (gramas/ dia) foi semelhante $(p=0,57)$ nos lactentes com constipação (mediana=9,0g; percentis 25 e 75: 6,9-13,1g) e naqueles com hábito intestinal normal (mediana $=8,8$; percentis 25 e $75: 6,1-12,9 \mathrm{~g}$ ).

Conclusões: $o$ consumo de fibra alimentar por crianças menores de dois anos foi semelhante entre os grupos com e sem constipação intestinal. $\mathrm{O}$ aleitamento natural predominante é fator de proteção contra constipação no primeiro semestre de vida.
\end{abstract}

J Pediatr (Rio J) 2002; 78 (3): 202-08: constipação, aleitamento materno, fibra na dieta.

1. Mestre em Nutrição pela Universidade Federal de São Paulo - Escola Paulista de Medicina.

2. Nutricionista, Doutora da Disciplina de Nutrição e Metabolismo do Dep. Pediatria da Univ. Federal de São Paulo - Escola Paulista de Medicina.

3. Professora Adjunta - Doutora da Disciplina de Pediatria Geral e Comunitária do Dep. de Pediatria da Univ. Federal de São Paulo - Escola Paulista de Medicina.

4. Professor Adjunto - Livre Docente e Chefe da Disciplina de Gastroenterologia Pediátrica do Dep. de Pediatria da Universidade Federal de São Paulo - Escola Paulista de Medicina.

Artigo submetido em 05.10.01, aceito em 27.03.02.

\begin{abstract}
Objective: To study the relationship between breast-feeding, dietary fiber intake and constipation in infants.

Methods: The study population consisted of 275 infants consecutively enrolled in two Primary Care Clinic in the city of Embu, in the Great São Paulo. The feeding pattern were classified in predominantly breast-feeding, partially breast and cow's milk feeding and artificial feeding. Constipation was defined by the elimination of hard stool associated with one of the following: painful or difficulty defecation, scybalous or cylindrical cracked stools and less than three defecations a week. False constipation was defined by the elimination of soft stools without pain or difficulty but with less than three defecations a week.
\end{abstract}

Results: Constipation was found in $25.1 \%$ (69/275). False constipation was found only in the first semester of life in $5.1 \%$ of 159 infants. The prevalence of constipation was higher between 6 and 24 months $(38.8 \%, 45 / 116)$ than in the first semester of life $(15.1 \%, \mathrm{P}=0.000)$. A model of logistic regression demonstrated that infants under artificial feeding were 4.53 times more liable to develop constipation than infants who were predominantly breastfed. The daily dietary fiber intake ( $\mathrm{g} /$ day) was similar $(\mathrm{P}=0.57)$ among the constipated (median $=9.0 \mathrm{~g} ; 25$ th and 75 th percentiles: $6.9-13.1 \mathrm{~g}$ ) and non-constipated (median $=8.8 \mathrm{~g} ; 25$ th and 75 th percentiles: 6.1 $12.9 \mathrm{~g})$.

Conclusions: Dietary fiber intake was similar in constipated and non-constipated infants. Breast-feeding serves as a protection factor against the development of constipation in the first semester of life.

J Pediatr (Rio J) 2002; 78 (3): 202-08: constipation, breastfeeding, dietary fiber.

\section{Introdução}

A etiopatogenia da constipação crônica funcional na criança não é plenamente conhecida. Acredita-se que fatores constitucionais, hereditários, alimentares e psicológicos ou emocionais, associados ou não a um distúrbio da motilidade intestinal, possam estar envolvidos na sua gêne$\mathrm{se}^{1-3}$. 
Estudos realizados em clínicas de gastroenterologia pediátrica, no Brasil ${ }^{4,5}$ e no exterior 6 , concordam que, freqüentemente, a constipação tem início precoce, muitas vezes ainda no primeiro semestre de vida ou, até mesmo, desde o nascimento. Em dois estudos ${ }^{4,5}$ realizados no Brasil, com pacientes atendidos em ambulatórios especializados, a maior parte com idade entre quatro e seis anos, observou-se que cerca de $20 \%$ dos responsáveis referiam que a constipação havia se iniciado na vigência do aleitamento natural exclusivo. Por outro lado, questiona-se a possibilidade de início da constipação na vigência de aleitamento natural exclusivo ${ }^{7}$. Em estudo anterior ${ }^{5}$, observamos, ainda, que a mediana do tempo de aleitamento natural exclusivo foi menor nas crianças com constipação em relação ao grupo controle, motivando a especulação de que o aleitamento natural pudesse desempenhar algum papel protetor no desenvolvimento da constipação. No entanto, essa conjectura deve ser analisada com prudência, em vista do longo intervalo entre a aplicação do questionário e a ocorrência do desmame ${ }^{5}$.

Na criança, dieta com baixa quantidade de fibra alimentar constitui fator de risco para constipação crônica ${ }^{8,9}$. Não existe na literatura, em nosso conhecimento, nenhum estudo relacionando o hábito intestinal com a quantidade de fibra alimentar na dieta de lactentes.

Pelo exposto, foi realizado o presente estudo, com o objetivo de estudar a relação entre o tipo de aleitamento, o consumo de fibra alimentar e a ocorrência de constipação em lactentes.

\section{Métodos}

O estudo foi realizado em duas unidades básicas de saúde no município de Embu, na Região Metropolitana de São Paulo. A Universidade Federal de São Paulo - Escola Paulista de Medicina (UNIFESP-EPM) - atua nesse município, num programa de integração docente assistencial, desde a década de 1970. Foram incluídas no estudo todas as crianças com idade inferior a 24 meses, atendidas consecutivamente no período de dezembro de 1996 a dezembro de 1997, desde que não apresentassem doenças agudas ou crônicas. Foram considerados como critérios de exclusão: presença de retardo do desenvolvimento neuropsicomotor, hipotiroidismo, malformações anorretais e uso de antiácidos, anticolinérgicos e codeína. Vale assinalar que lactentes com essas características não foram encontrados. Com esta sistemática, foram incluídos no estudo 275 lactentes.

Para avaliar se a criança era portadora ou não de constipação, foi utilizado um questionário padronizado, com questões relativas às características mais freqüentes do hábito intestinal. Registrou-se a forma, a consistência das fezes e a ocorrência de dor, dificuldade ou medo durante as evacuações. Para auxiliar na obtenção de informação da forma e consistência predominante das fezes, foi utilizado um catálogo com 12 fotografias de fezes de lactentes com diferentes características.
Constipação foi caracterizada quando havia eliminação de fezes com consistência obrigatoriamente dura e a ocorrência de pelo menos uma das seguintes características: dor ou dificuldade ao evacuar, fezes com formato de cíbalos, cilíndrica com rachaduras ou cilíndrica e espessa, ou, ainda, quando o intervalo entre as evacuações era maior ou igual a três dias. Pseudoconstipação foi caracterizada quando ocorria a eliminação de fezes amolecidas, sem dor ou dificuldade, em intervalo maior ou igual a três dias ${ }^{3}$. Hábito intestinal normal foi definido quando o lactente não apresentasse as características expostas acima.

Quanto à prática de aleitamento materno, os lactentes foram distribuídos em três categorias ${ }^{10}$ :

- aleitamento predominante: lactentes que recebiam exclusivamente aleitamento materno ou que recebiam aleitamento materno, água e/ou chá;

- aleitamento misto: quando a criança recebia leite materno e outros alimentos complementares, que poderiam ser leite de vaca, fórmula láctea ou qualquer outro alimento; e

- aleitamento artificial: quando a criança não recebia mais leite materno.

Para os lactentes com idade superior a seis meses, foi avaliado o consumo de alimentos pelo método do dia alimentar habitual ${ }^{11}$. A análise das informações da dieta foram realizadas com o programa Apoio à Nutrição ${ }^{12}$. A quantidade de fibra alimentar foi avaliada utilizando a tabela americana da AOAC (Association of Official Analytical Chemists) ${ }^{13}$.

As medidas do peso e da estatura foram obtidas segundo as técnicas recomendadas ${ }^{14}$. A comparação entre os parâmetros antropométricos das crianças dos grupos com constipação e controle foi realizada por meio dos escores z, calculados pelo programa EPI-Info ${ }^{15}$, em relação às tabelas de peso e estatura do NCHS (National Center for Health Statistics $)^{16}$.

A análise estatística foi realizada com o emprego do programa EPI-Info ${ }^{15}$. Para a análise multivariada do desfecho constipação, foi utilizado modelo de regressão logística, com o emprego do programa Stata (Stata Corporation, Texas, USA $)^{17}$. O projeto foi aprovado pela Comissão de Ética da Universidade Federal de São Paulo - Escola Paulista de Medicina, sendo obtido consentimento por escrito dos responsáveis.

\section{Resultados}

De acordo com a faixa etária, os lactentes foram distribuídos em duas categorias: menores de 6 meses, e de 6 a 24 meses de vida. Na Tabela 1, é apresentado o padrão do hábito intestinal e a ocorrência ou não de constipação. A ocorrência de constipação na população estudada foi igual a $25,1 \%$ (69/275). A forma das fezes em cíbalos foi a mais freqüente nos lactentes com constipação, ocorrendo em 63,4\% (44/69) dos casos. Freqüência de evacuações inferi- 
or a três por semana foi observada em apenas 5,8\% dos 69 lactentes com constipação. Constipação foi mais freqüente entre os 6 e 24 meses $(38,8 \%$; 45/116) do que nos primeiros seis meses de vida $(15,1 \%)$, sendo a diferença estatisticamente significante $(\mathrm{p}=0,0000)$. Quanto à percepção da mãe sobre a ocorrência de dor e/ou dificuldade durante as evacuações de seus filhos, observou-se que esteve presente em $65,2 \%(15 / 23)$ e $25,2 \%$ (30/119) dos lactentes com constipação, respectivamente, nos primeiros 6 meses de vida e entre 6 e 24 meses. Por outro lado, dentre os lactentes com hábito intestinal normal, a ocorrência de dor e/ou dificuldade esteve presente em $25,2 \%$ (30/119) nos primeiros 6 meses de vida, e em nenhum dos lactentes entre 6 e 24 meses.

A relação entre tipo de aleitamento e ocorrência de constipação foi analisada considerando-se as informações obtidas com 159 lactentes, no primeiro semestre de vida. Nesse grupo, $66(41,5 \%)$ recebiam aleitamento natural predominante, $49(30,8 \%)$, aleitamento natural misto e 44 $(27,7 \%)$, aleitamento artificial. Observou-se que $8(5,1 \%)$ dos 159 apresentavam pseudoconstipação, ou seja, eliminação de fezes amorfas, com consistência líquida ou pasto$\mathrm{sa}$, em intervalo maior do que 3 dias, sendo que 6 recebiam aleitamento natural predominante, e 2 aleitamento natural misto. A freqüência de evacuações foi igual a 2 vezes por semana em 4 lactentes com pseudoconstipação, 1 vez por semana em três, e uma vez a cada 15 dias em um lactente.
A ocorrência de constipação foi menor nos lactentes que recebiam aleitamento natural predominante $(4,5 \%) \mathrm{em}$ relação aos que recebiam aleitamento misto $(18,4 \%)$ ou artificial $(27,3 \% ; 12 / 44 ; \mathrm{p}<0,05)$. Observou-se, também, dentro do primeiro semestre de vida, aumento da ocorrência de constipação com o aumento da idade (5,5\% no primeiro bimestre, $16,3 \%$ no segundo, e 23,0\% no terceiro; $p<0,05$ ). Levando em consideração que, com o aumento da idade, ocorreu aumento na frequiência de constipação e, também, diminuição na freqüência de aleitamento predominante; a relação entre essas duas variáveis e a ocorrência de constipação foi analisada por modelo de regressão logística, considerando três categorias para a idade (primeiro, segundo e terceiro bimestres de vida) e para o tipo de aleitamento (predominante, misto e artificial). Na Tabela 2, é apresentado o resultado da análise de regressão logística, mostrando que a idade é um fator não associado à constipação, ao contrário do indicado pelo teste estatístico usado na avaliação da associação dessas variáveis de forma isolada. Por outro lado, foi confirmada a associação entre tipo de aleitamento e constipação $(\mathrm{p}=0,02)$. Assim, de acordo com o odds ratio, confirmou-se que o aleitamento artificial em relação ao aleitamento misto, e que o aleitamento misto em relação ao predominante, apresentaram chance proporcional 2,1 vezes maior do lactente apresentar constipação. Por sua vez, o aleitamento artificial em relação ao aleitamento natural predominante apresentou 4,5 vezes mais chance do lactente apresentar constipação.

Tabela 1 - Padrões do hábito intestinal de lactentes atendidos em unidade básica de saúde

\begin{tabular}{|c|c|c|c|c|c|c|}
\hline \multicolumn{2}{|l|}{ Fezes } & \multirow{2}{*}{$\begin{array}{c}\text { Dor e/ou } \\
\text { dificuldade } \\
\text { nas evacuações }\end{array}$} & \multirow[b]{2}{*}{ Conclusão } & \multicolumn{3}{|c|}{ Faixa etária } \\
\hline Consistência & Forma & & & $0 \mid-6 \mathbf{m}$ & $6 \mid-24 m$ & Total \\
\hline Líquida, mole ou pastosa & amorfa & Não & Normal & $83(52,2 \%)$ & $40(34,5 \%)$ & $123(44,7 \%)$ \\
\hline Líquida, mole ou pastosa & amorfa & Sim & Normal & $29(18,2 \%)$ & $0(0,0 \%)$ & $29(10,5 \%)$ \\
\hline Líquida, mole ou pastosa & cilíndrica & Não & Normal & $6(3,7 \%)$ & $16(13,7 \%)$ & $22(8,0 \%)$ \\
\hline Líquida, mole ou pastosa & cilíndrica & Sim & Normal & $1(0,6 \%)$ & $0(0,0 \%)$ & $1(0,4 \%)$ \\
\hline Dura & $\begin{array}{c}\text { cilíndrica } \\
\text { sem rachadura }\end{array}$ & Não & Normal & $8(5,0 \%)$ & $15(12,9 \%)$ & $23(8,4 \%)$ \\
\hline Dura & cíbalo & Sim & Constipação & $11(6,9 \%)$ & $18(15,5 \%)$ & $29(10,5 \%)$ \\
\hline Dura & cíbalo & Não & Constipação & $4(2,5 \%)$ & $11(9,4 \%)$ & $15(5,5 \%)$ \\
\hline Dura & $\begin{array}{c}\text { cilíndrica } \\
\text { sem rachadura }\end{array}$ & Sim & Constipação & $2(1,2 \%)$ & $6(5,1 \%)$ & $8(2,9 \%)$ \\
\hline Dura & $\begin{array}{l}\text { cilíndrica } \\
\text { com rachadura }\end{array}$ & Sim & Constipação & $3(1,89 \%)$ & $5(4,3 \%)$ & $8(2,9 \%)$ \\
\hline Dura & $\begin{array}{l}\text { cilíndrica } \\
\text { com rachadura }\end{array}$ & Não & Constipação & $4(2,5 \%)$ & $5(4,3 \%)$ & $9(3,3 \%)$ \\
\hline \multicolumn{2}{|c|}{$\begin{array}{l}\text { Fezes líquidas, moles ou pastosas eliminadas } \\
\text { com intervalo maior ou igual a } 3 \text { dias }\end{array}$} & & $\begin{array}{c}\text { Pseudo-constipação } \\
\text { Total }\end{array}$ & $\begin{array}{c}8(5,0 \%) \\
159(100,0 \%)\end{array}$ & $\begin{array}{c}0(0,0 \%) \\
116(100,0 \%)\end{array}$ & $\begin{array}{c}8(2,9 \%) \\
275(100,0 \%)\end{array}$ \\
\hline
\end{tabular}


Tabela 2 - Resultado da análise de regressão logística com categorias adjacentes proporcionais sobre a relação entre constipação, idade e tipo de aleitamento em 159 lactentes no primeiro semestre de vida

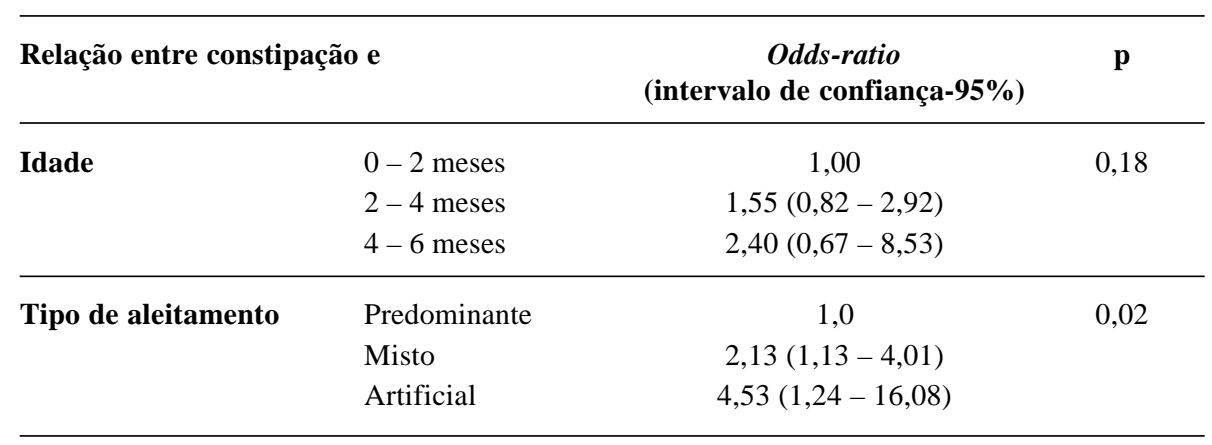

O estudo da relação entre consumo de nutrientes, de outras informações da dieta e de fibras alimentares foi realizada nos lactentes com idade superior a seis meses. $\mathrm{O}$ consumo de alimentos foi avaliado de acordo com o inquérito do dia alimentar habitual, com as informações obtidas sobre 75 lactentes com idade superior a 6 meses, que não recebiam mais leite materno. Foi necessário excluir seis $(8,0 \%)$ inquéritos alimentares com informações inadequa- das. Assim, os resultados apresentados na Tabela 3 correspondem a 31 crianças com constipação, e a 38 com hábito intestinal normal. A idade e as estimativas das quantidades de alimentos, dos volumes de água e de leite, das adequações energética e protéica e das quantidades de proteína, carboidratos e lipídeos não apresentaram diferença estatisticamente significante entre as crianças com e sem constipação.

Tabela 3 - Idade (meses) e dados da dieta segundo o dia alimentar habitual de lactentes com ou sem constipação

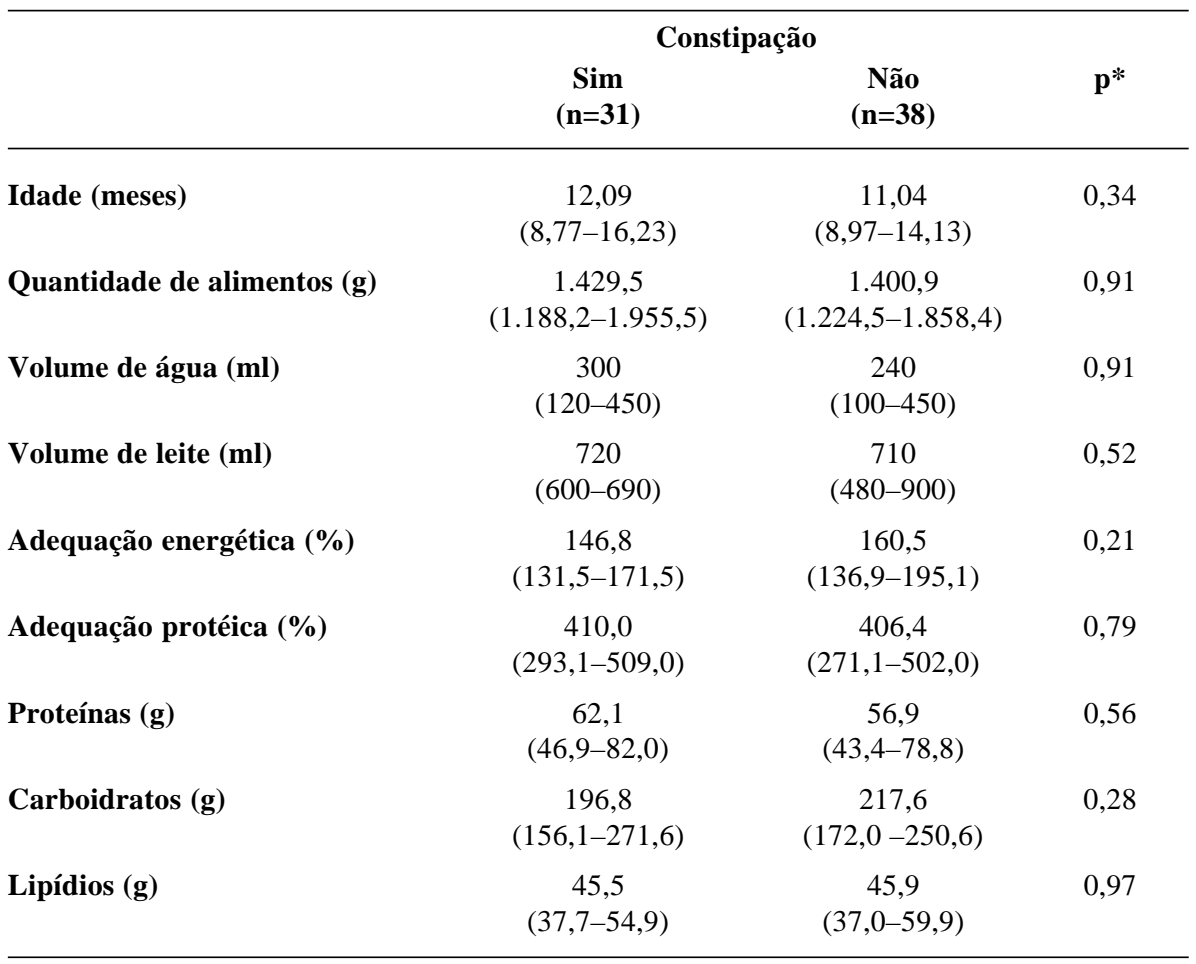

* Mediana e percentis 25 e 75, teste de Mann-Whitney para todas as variáveis. 
A estimativa do consumo de fibra alimentar foi realizada de acordo com a tabela da AOAC. Nessa avaliação foram incluídas, também, as crianças que recebiam leite materno, que não é fonte de fibra alimentar. Dentre os inquéritos alimentares das 116 crianças com idade entre 6 e 24 meses, foram excluídos $9(7,8 \%)$ inquéritos que não apresentavam informações adequadas. Assim, foram considerados os resultados de 107 inquéritos alimentares. A Tabela 4 mostra que não houve diferença estatística no consumo de fibra alimentar pelas crianças com constipação em comparação com as crianças sem constipação.

Na Tabela 5, são apresentados os parâmetros antropométricos, que não apresentaram diferença estatística segundo a ocorrência ou não de constipação.

\section{Discussão}

No Brasil, vários estudos revistos recentemente ${ }^{3}$ mostraram que constipação ocorre com elevada frequiência na população pediátrica, acrescentando-se nosso resultado de $25,1 \%$ em menores de 2 anos, faixa etária pouco explorada nas publicações anteriores. Neste estudo, verificou-se que a ocorrência de constipação aumenta, progressivamente, durante o primeiro semestre da vida. Por sua vez, a prevalência de constipação entre os 6 e 24 meses é estatisticamente superior à observada nos primeiros 6 meses de vida. Estudo ${ }^{18}$ realizado em comunidade do Nordeste do Brasil mostrou prevalência de constipação em lactentes igual a $21,8 \%$, valor semelhante ao resultado do presente estudo. Vale mencionar que é difícil comparar os resultados dos diferentes estudos sobre ocorrência de constipação devido às diferenças nos conceitos adotados nos diferentes estudos. No presente estudo, constipação foi caracterizada quando havia eliminação de fezes com consistência obrigatoriamente dura e a ocorrência de pelo menos mais de uma das seguintes características: dor ou dificuldade ao evacuar, fezes com formato de cíbalos ou cilíndrica, com rachaduras, ou, ainda, quando o intervalo entre as evacuações era maior ou igual a 3 dias. Este conceito é concordante com a proposta de um grupo de trabalho com representantes dos diferentes continentes do mundo ${ }^{19}$. Nossos resultados mostraram que, em lactentes, a constipação se apresentou, em geral, com fezes duras, na forma de cíbalos, acompanhadas de dor ou de dificuldade durante as evacuações. Diminuição na frequiência de evacuações ocorreu em apenas 5,8\% dos lactentes com constipação. Por outro lado, menção de dor e/ou dificuldade durante as evacuações foi relatada por $25,2 \%$ das mães de lactentes com menos de 6 meses de

Tabela 5 - Mediana e percentis 25 e 75 (entre parênteses) dos escores z de peso para idade, peso para estatura e estatura para idade das crianças com e sem constipação

\begin{tabular}{|c|c|c|c|}
\hline \multicolumn{4}{|c|}{ Constipação } \\
\hline Escore z & $\underset{(n=69)}{\operatorname{Sim}}$ & $\begin{array}{c}\text { Não } \\
(\mathbf{n}=\mathbf{2 0 6})\end{array}$ & $\begin{array}{c}\text { Teste de } \\
\text { Mann-Whitney }\end{array}$ \\
\hline
\end{tabular}

$\begin{array}{lccc}\begin{array}{l}\text { Peso/idade } \\ \text { Mediana } \\ \text { (P25 P75) }\end{array} & 0,0 & 0,0 & 0,51 \\ \begin{array}{l}\text { Peso/estatura } \\ \text { Mediana } \\ \text { (P25 P75) }\end{array} & \begin{array}{c} \\ (-0,7+0,6)\end{array} & (-0,6+0,5) & \\ \begin{array}{l}\text { Estatura/idade } \\ \text { Mediana } \\ \text { (P25 P75) }\end{array} & \begin{array}{l} \\ \end{array} & (-0,4+0,2 & 0,12 \\ & (-1,0+0,4) & -0,3 & \\ & & (-0,9+0,3) & 0,77\end{array}$

Obs: os escores $z$ do peso para a estatura foram calculados para 68 crianças com constipação e 203 sem constipação, uma vez que quatro crianças apresentaram estatura abaixo do menor valor da tabela do NCHS.

Tabela 4 - Estimativa do consumo de fibra alimentar total (g), adequação percentual ao recomendado (5 gramas/dia) e fibra alimentar por 1.000 kcalorias de dieta segundo o dia alimentar habitual de lactentes com ou sem constipação

\begin{tabular}{lccc}
\hline & \multicolumn{2}{c}{ Constipação } & Sim \\
& $(\mathbf{n = 4 4})$ & $\begin{array}{c}\text { Não } \\
(\mathbf{n}=\mathbf{6 3})\end{array}$ & p* \\
\hline Consumo diário (gramas/dia) & 9,00 & 8,76 & 0,57 \\
& $(6,88-13,13)$ & $(6,07-12,94)$ & \\
$\begin{array}{l}\text { Percentual da recomendação mínima } \\
\text { (5 gramas por dia) }\end{array}$ & $180,0 \%$ & $175,2 \%$ & 0,57 \\
$\begin{array}{l}\text { Fibra total (gramas) por } \\
1.000 \text { kcal da dieta }\end{array}$ & $(137,6 \%-262,6 \%)$ & $(121,4 \%-258,8 \%)$ & \\
\hline
\end{tabular}

* Mediana e percentis 25 e 75 entre parênteses, teste de Mann-Whitney para todas as variáveis. 
idade, que não apresentavam constipação, mostrando que, nessa faixa etária, esse comemorativo é pouco específico. Quanto à pseudoconstipação, não há referências na literatura sobre sua freqüência, que foi de 5,1\% nos 159 lactentes com menos de 6 meses de idade, que correspondiam a 7,3\% (8/110) dos lactentes que se encontravam em aleitamento natural predominante ou misto. Deve ser ressaltado que a pseudoconstipação não é uma condição patológica e, portanto, não necessita receber qualquer tipo de tratamento, exceto orientação para os familiares e, evidentemente, manutenção do aleitamento natural. Vale lembrar que os lactentes constipados incluídos no presente estudo não estavam recebendo tratamento específico. Esse aspecto se contrapõe ao observado nos ambulatórios de gastroenterologia pediátrica, onde, em geral, são atendidas crianças com maior idade e quadros graves, nos quais a constipação constitui a queixa principal. Nesses ambulatórios especializados, é comum encontrar as complicações da constipação, que motivam a assistência especializada, entre as quais o escape fecal (soiling), o comportamento de retenção, a eliminação muito dolorosa e esporádica de fezes muito volumosas e dor abdominal crônica ${ }^{4-6}$. Vale reiterar que, nesses pacientes com constipação crônica, atendidos em serviços especializados, freqüentemente observa-se início do problema no primeiro ano de vida, razão pela qual investigamos, em lactentes, a relação entre constipação e dois aspectos da dieta que podem estar relacionados com o seu início: tipo de aleitamento e quantidade de fibra alimentar na dieta de desmame.

No primeiro semestre de vida, constatou-se que a ausência do aleitamento natural é um fator associado com constipação, segundo o teste de regressão logística. Em outras palavras, a probabilidade de constipação entre crianças com aleitamento artificial é 4,5 vezes maior do que entre lactentes em aleitamento predominante. Segundo a literatu$\mathrm{ra}^{20,21}$, o número de evacuações é maior no primeiro ano de vida, e diminui com o aumento da idade. A frequiência de evacuações em lactentes em aleitamento natural é maior do que a dos em aleitamento artificial. A motilina pode ser um dos responsáveis pela diferença no hábito intestinal ${ }^{20}$. Outro aspecto relaciona-se com a boa digestibilidade dos lipídeos do leite humano em relação às fórmulas, o que favorece a formação de fezes menos consistentes ${ }^{22}$. Após o desmame, alguns casos de constipação podem ser atribuídos à intolerância ou alergia à proteína do leite de vaca ${ }^{23-25}$. Acrescentando-se essas evidências fisiológicas ${ }^{20,21}$ e patológicas $^{21-23}$ aos resultados do nosso estudo, o aleitamento natural deve ser considerado como um fator de proteção contra o desenvolvimento de constipação.

Outro fator da dieta que pode influenciar no desenvolvimento de constipação no lactente é o teor de fibra alimentar nos alimentos de desmame. No entanto, não existe trabalho na literatura que relacione o consumo de fibra alimentar com o hábito intestinal nesta faixa etária. As informações sobre o consumo de fibra alimentar em lactentes são escassas. Existem preocupações de que a alta inges- tão de fibra alimentar possa ter repercussões negativas no crescimento, por provocar saciedade precoce, ou por interferência na absorção de minerais. Quanto ao consumo de fibra alimentar por lactentes, a única recomendação encontrada na literatura foi proposta por Agostoni et al. ${ }^{26}$, sugerindo consumo diário de pelo menos 5 gramas de fibra alimentar por dia. No presente estudo, não se observou associação entre constipação e menor ingestão de fibras, diferenciando-se dos resultados de outros estudos realizados em nosso país ${ }^{8}$ e no exterior ${ }^{9}$, nos quais foram estudadas crianças em faixa etária superior aos 2 anos.

Apesar de ter sido referido na literatura ${ }^{27-30}$ que uma alta ingestão de fibra alimentar pode provocar anorexia, interferindo, assim, na quantidade de energia consumida, em nosso estudo os lactentes apresentavam consumo mediano de energia e de fibra maior do que a recomendação mínima, e os valores de peso e estatura demonstraram que a população estudada apresentava condição nutricional satisfatória. É importante ressaltar que o fato de as crianças com e sem constipação não apresentarem diferentes estimativas de consumo de fibras não significa que a administração de maior quantidade de fibras para aquelas com tendência à constipação não ocasione normalização do hábito intestinal.

Em conclusão, nosso estudo mostrou que a estimativa de consumo de fibra alimentar não foi diferente nos lactentes com e sem constipação. Quanto ao aleitamento natural, pode-se afirmar que às suas diversas vantagens em relação ao aleitamento artificial deve ser acrescentado o papel protetor contra o desenvolvimento de constipação no primeiro semestre de vida.

\section{Agradecimentos}

Agradecemos ao apoio da Fundação de Amparo à Pesquisa de São Paulo (FAPESP), e também a Fernando A.B. Colugnati, pela análise estatística e pela análise do teste de regressão logística.

\section{Referências bibliográficas}

1. Clayden G. Management of cronic constipation. Arch Dis Child 1992; 67:340-4.

2. Loening-Baucke V. Chronic constipation in children. Gastroenterology 1993; 105:1557-64.

3. Morais MB, Maffei HVL. Constipação em pediatria. J Pediatr (Rio J) 2000; 76(Sup12): S147-S156.

4. Maffei HVL, Moreira FL, Kissimoto M, Chaves SM, Elfaro S, Aleixo AM. História clínica e alimentar de crianças atendidas em ambulatório de gastroenterologia pediátrica com constipação intestinal crônica funcional e suas possíveis complicações. J Pediatr (Rio J) 1994; 70:280-6. 
5. Morais MB, Vitolo MR, Aguirre ANC, Medeiros EHGR, Antonelli EMAL, Fagundes-Neto U. Teor de fibra alimentar e de outros nutrientes na dieta de crianças com e sem constipação crônica funcional. Arquivos de Gastroenterol 1996; 33:93-101.

6. Loening-Baucke V. Encopresis and soiling. Pediatr Clin North Am 1996; 43:279-98.

7. Murahovschi J, Teruya KM. [Carta ao editor]. J Pediatr (Rio J) 1995; 71:101-2.

8. Morais MB, Vítolo MR, Aguirre ANC, Fagundes-Neto U. Measurement of low dietary fiber intake as a risk factor for chronic constipation in children. J Pediat Gastroenterol Nutr 1999; 29:132-5.

9. Roma E, Adamidis D, Nikolara R, Constantopoulos A, Messaritakis J. Diet and chronic constipation in children: the role of fiber. J Pediatr Gastroenterol Nutr 1999; 28:169-74.

10. Organizacion Panamericana de la Salud, Organizacion Mundial de la Salud. Indicadores para evaluar las practicas de lactancia materna. Ginebra: OMS; 1991.

11. Thompson FE, Byers T. Dietary assessment resource manual. J Nutr 1994; 124:2245S-301S.

12. Anção M. Centro de Informática da Unifesp-EPM. Sistema de Apoio à Nutrição, versão 2,5, 1995.

13. Shils M, Olson S, Shike M. Modern nutrition in health and diseases. $8^{\mathrm{a}}$ ed. Philadelphia: Lea e Febiger; 1994.p.A92-A98.

14. Jelliffe DB. Evaluación del estado de nutrición de la comunidad. Genebra: Organización Mundial de la Salud (Série de monografias 53); 1968

15. Dean AG, Dean JA, Coulombier D, Brendel KA, Smith DC, Burton AH, et al. Epi-Info, Version 6.0: a word processor database and statistics program for epidemiology on microcomputers. Atlanta, Georgia: Center of Disease Control and Prevention; 1994.

16. National Center for Health Statistics - Growth curves for children birth-17 years. United States Department of Health Education and Welfare. Vital and Health Statistics. Series 11, Nb. 165. Hyattsville, MD: DHEW publication (PHS); 1977.p.78-1650.

17. Software Estatístico Stata. Texas, USA: Stata Corporation.

18. Motta MEFA, Silva GAP. Constipação intestinal crônica funcional na infância: diagnóstico e prevalência em uma comunidade de baixa renda. J Pediatr (Rio J) 1998; 74:451-4.

19. Hyams J, Colletti R, Morais MB, Faure C, Gabriel-Martinez E, Maffei HVL, et al. Functional gastrointestinal disorders. Report of the working groups 2000: A global plan for the future of the digestive and nutritional health of children. Boston, World Congress of Pediatric Gastroenterology, Hepatology \& Nutrition; 2000.p.83-94.
20. Weaver LT, Ewing G, Taylor LT. The bowel habit of milk-fed infants. J Pediatr Gastroenterol Nutr 1988; 7:568-71.

21. Weaver LT. Bowel habit from birth to old age. J Pediatr Gastroenterol Nutr 1988; 7:637-40.

22. Quinlan PT, Lockton S, Lucas AL. The relationship between stool hardness and stool composition in breast- and formula-fed infants. J Pediatr Gastroenterol Nutr 1995; 20:81-90.

23. Iacono G, Cavatio F, Montalto G, Florena MD, Tumminello M, Soresi M, et al. Intolerance of cow's milk and chronic constipation in children. N Engl J Med 1998; 339:1100-4.

24. Shan N, Lindley K, Milla P. Cow's milk and chronic constipation in children [correspondence]. N Engl J Med 1999; 340:891-2.

25. Daher S, Solé D, Morais MB. Cow's milk intolerance and chronic constipation in children [correspondence]. NEngl J Med 1999; 340:891-2.

26. Agostoni C, Riva E, Giovannini M. Dietary fiber in weaning foods of young children. Pediatrics 1995; 96:1002-5.

27. Dwyer JT. Dietary fiber for children: how much? Pediatrics 1995; 96:1019-22.

28. Dagnelie PC, Van Staveren WA, Verschuren SAJM, Hautvast JGAJ. Nutritional status of infants aged 4 to 18 months on macrobiotic diets and matched omnivorous control infants: a population-based mixed-longitudinal study I. Weaning pattern, energy and nutrient intake. Eur J Clin Nutr 1989; 43:311-23.

29. Araya H, Vera G, Alviña M, Fuentes A, Oyarzun MT, Pak N. Efecto de diferentes niveles de almidón y fibra dietética de preparaciones sobre el consumo inmediato y subsecuente de preescolares de 24 a 48 meses de edad. Arch Latinoam Nutr 1994; 44:12-7.

30. Dagnelie PC, Van Staveren WA. Macrobiotic nutrition and child health: results of a population- based, mixed- longitudinal cohort study in the Netherlands. Am J Clin Nutr 1994; 59:1187S- 96S.

Endereço para correspondência:

Dra. Andrea Nogueira de Campos Aguirre

Rua Coronel Amazonas Marcondes, 1115 - apto. 203 C2

CEP 80035-230 - Curitiba, PR

Fone: (41) 353.4839

E-mail: andrea.aguirre@utp.br 